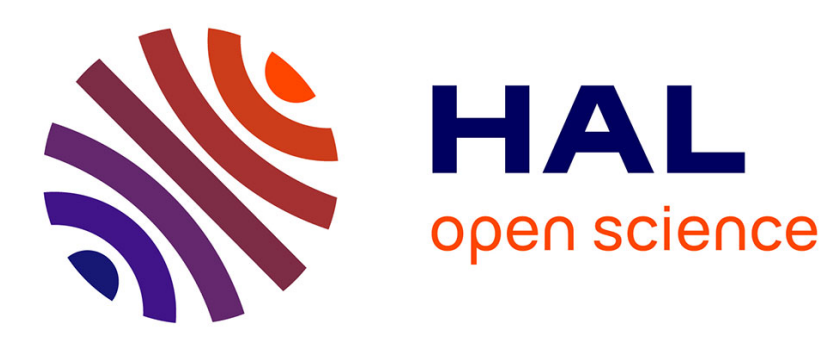

\title{
Light angular momentum of a plane wave diffracted by a two-dimensional simple object.
}

Olivier Emile, Morgane Le Meur, Janine Emile

\section{To cite this version:}

Olivier Emile, Morgane Le Meur, Janine Emile. Light angular momentum of a plane wave diffracted by a two-dimensional simple object.. Physical Review A: Atomic, molecular, and optical physics [1990-2015], 2014, 89 (1), pp.013846. 10.1103/PhysRevA.89.013846 . hal-00993185

\section{HAL Id: hal-00993185 \\ https://hal.science/hal-00993185}

Submitted on 19 May 2014

HAL is a multi-disciplinary open access archive for the deposit and dissemination of scientific research documents, whether they are published or not. The documents may come from teaching and research institutions in France or abroad, or from public or private research centers.
L'archive ouverte pluridisciplinaire HAL, est destinée au dépôt et à la diffusion de documents scientifiques de niveau recherche, publiés ou non, émanant des établissements d'enseignement et de recherche français ou étrangers, des laboratoires publics ou privés. 


\title{
Light angular momentum of a plane wave diffracted by a two-dimensional simple object.
}

\author{
Olivier Emile, ${ }^{1}$ Morgane Le Meur, ${ }^{1}$ and Janine Emile ${ }^{2}$ \\ ${ }^{1}$ LPL, URU 435 Université de Rennes I, 35042 Rennes Cedex, France.* \\ ${ }^{2}$ IPR, UMR CNRS 6251, Université de Rennes I, 35042 Rennes Cedex, France.
}

(Dated: December 9, 2013)

\begin{abstract}
The question of the transfer of angular momentum from light to objects is a key issue in optical tweezers and thus raises issues in physics, chemistry and biology. Most of the attempts have been performed either with spin angular momentum using light's polarization or using orbital angular momentum with twisted beams. We experimentally demonstrate here the exchange of angular momentum from a linearly polarized plane wave to a two dimensional asymmetric object. More precisely, we show that the diffracted light from a snail-shaped mask, carries orbital angular momentum which topological charge depends on the symmetry of the object. This opens the way to new means to produce micrometer-sized twisted beams from high power laser or even pulsed beams.
\end{abstract}

PACS numbers: 42.50.Tx, 03.65.Vf, 42.87.-d, 42.25.Fx 
Since 1992, there has been a revival of interest for the angular momentum of light [1-3]. Apart from fundamental considerations, they are many potential applications as, for example, in astrophysics [4], microwaves [5], atom optics [6, 7], rotation of particles $[8,9]$, manipulations of objects of biological interest $[10,11]$, telecommunications [12, 13], quantum entanglement [14, 15], acoustics [16], or electron beams [17]. These beams are generally produced either from dedicated optical elements $[1,18,19]$, or from the diffraction of particular gratings [20-22]. Such beams have been recently shown to be able to interfere in the shadow of a circular disk, thus generating non-diffracting beams with micro-meter dimensions [23]. In particular, since the disk is symmetrical, the beam in its shadow keeps the same topological charge as the incident beam. On the other hand, one may seek for new, simple, and effective ways to produce twisted beams with micrometer size in the context for example of atom guiding [24, 25] or for broadband sources that can be adapted to high power continuous, pulsed light beams, or white light sources. One may then wonder what would the diffracted light look like in the shadow of a simple asymmetrical disk, illuminated by a single plane wave. The aim of this work is to investigate such diffracted light, and to look for possible connexions with twisted beams.

Let us consider the experimental set-up depicted in figure 1. A collimated red laser beam $(\lambda=633 \mathrm{~nm}$ ) illuminates a $2 \mathrm{~mm}$ diameter two-dimensional (2D) object. We have used four different objects, (i) a circular disk that is fully symmetrical under rotation. (ii) a snail shaped 2D object or single cam (wheel used in mechanics) with one notch that has a $2 \pi / l(l=1)$ symmetry, we will call it a $l=1$ object in the following. (iii) a double snail or double cam with two notches that has a $2 \pi / l(l=2)$ symmetry, we will call it a $l=2$ object. (iv) a triple snail or triple cam with three notches that has a $2 \pi / l(l=3)$ symmetry, we will call it a $l=3$ object. By analogy, the symmetrical disk will be called a $l=0$ object. Actually, we show below that the $l$ object generates twisted beams with a $l$ topological charge. 
These objects are drawn with a computer and printed in black on a transparency. The diffracted beam in the shadow of the object is best observed on a screen, at a distance $d=6 \mathrm{~m}$, with the naked eye or with a camera. For a $l=0$ object, one recognizes on figure 1, the usual Arago-Poisson non-diffracting bright spot [26], as expected. Surprisingly, for $l=1,2,3$ objects, the Arago-Poisson bright spot is replaced by a small dark spot surrounded by bright light. It strongly resembles the dark spot recently observed [23] in the shadow of a perfect symmetrical disk that was illuminated by a twisted beam generated from a spiral phase plate. This suggests that a single plane wave diffracted by a simple asymmetric object may have the same spatial distribution as a twisted beam diffracted by a symmetrical object. It has already been shown that in the case of gratings, the wave generated by asymmetrical sources [5] or diffracted by asymmetrical gratings [20-22] is indeed twisted. However it has never been explored in the case of simple asymmetrical objects.

We have developed numerical simulations based on Fresnel diffraction [27], to investigate the structure of such diffracted beams. Using exactly the same masks as the ones printed for the experiments ( $1^{\text {st }}$ input parameter), we calculate the diffracted field from a plane wave, for a wavelength $\lambda\left(2^{d}\right.$ input parameter), at a distance $d$ ( $3^{d}$ input parameter) from the diffracting object. From the simulations, we can either extract the light intensity or the phase distribution of the light field. The comparison between our simulations and the experimental observations on the intensity of the diffracted beam shows a very good agreement (see figure 1).

We have experimentally changed the various input parameters, starting with the wavelength of the beam, using different lasers (see figure 2a). At a given distance $d$, where the image is clear, the size of the dark spot increases linearly with the wavelength, as usual for diffraction patterns $[23,26]$, for every value of $l$.

We have then changed the distance $d$ between the diffracting object and the screen. We have measured the size of the dark spot versus $d$ (see figure $2 \mathrm{~b}$ ), for 
$l=1,2,3$. One distinguishes four zones: zone1 $2 \mathrm{~m}<d<4 \mathrm{~m}$, zone2 $4 \mathrm{~m}<d<5 \mathrm{~m}$, zone3 $5 \mathrm{~m}<d<10 \mathrm{~m}$ and zone4 $d>10 \mathrm{~m}$. Zone2 seems to be a transition zone: the diffracted beam undergoes specific deformations and the dark spot is hardly noticeable. In zone4, the dark spot slowly disappears and evolves into the usual Arago-Poisson bright spot for $d>20 \mathrm{~m}$ (not shown in figure 2b). Let us thus focus on zone1 and zone3. We clearly identify a dark spot surrounded by bright light in the shadow of the disk. Its diameter increases linearly with the distance, as usual for diffraction patterns $[23,26]$. However, the slope of its variation is divided by a factor of two between zone1 and zone3. The straight line in zone1 for $l=1$, matches the straight line in zone3 for $l=2$, with a transition zone in between. Thus, it seems impossible to find an analytical expression describing the size of the dark spot for the different input parameters.

Finally, we have modified the shape of the diffracting object. Figures $2 \mathrm{a}$ and $2 \mathrm{~b}$ show that the diameter of the dark spot varies linearly with the number of notches $(l)$. We have checked that the observation of this dark spot is not specific to the dimensions of the cam we used. A smaller diameter of the cam or a smaller notch only shifts the various zones towards smaller $d$ values.

Are these diffracted beams actually twisted? Let us extract the phase of the beam from the simulations (figure 3). For $l=0$ one evidences a supraluminal propagation as already demonstrated [26], thus validating our phase simulations. For $l=1,2,3$ and $d=6 \mathrm{~m}$, the phase of the beam varies from 0 to $2 \pi l$ in one turn. The diffracted beam carries angular momentum with a topological charge equal to the number of notches $l$. However, for $d=3 \mathrm{~m}$, the phase of the diffracted beam varies from 0 to $4 \pi l$ in one turn. The diffracted beam thus carries angular momentum with a topological charge equal to $2 l$, confirming the differences observed between zone1 and zone3 in figure $2 \mathrm{~b}$. For $d>20 \mathrm{~m}$, we have checked that the phase is constant, then the topological charge equals zero. Since the topological charge of the diffracted beam varies from $2 l$ to 0 , the topological charge is not a 
conserved quantity along propagation.

Let us focus on the transition zone, zone2, in order to understand how the topological charge of the diffracted beam by a single snail changes from $l=2$ to $l=1$. We have simulated on Fig. 4 the phase variation of the beam, for various distances $d$ between the diffracting object and the observation, with a step of 0.25 $\mathrm{m}$. For $d=3 \mathrm{~m}$, the topological charge clearly equals 2 , and for $d=6 \mathrm{~m}$ the topological charge equals 1 . Actually, the phase distribution on a circle of $d=3$ with $4 \pi$ per turn slowly moves into a spiral $(d=4)$ that then starts to close $(d=5)$ with $2 \pi$ per turn. This explains why it was so difficult to evaluate the size of the dark spot since the beam has lost its cylindrical symmetry. The jump from $l=2$ to $l=1$ is not sudden. Since this diffracted beam is not an eigen solution of the equation of propagation, it thus could be that the topological charge is not conserved along propagation.

Actually, is it possible to experimentally evidence the twisted nature of the beam? Among the various existing techniques, we choose to measure the topological charge using the interference pattern from Young's double slit set-up [28]. We now use a green laser $(\lambda=532 \mathrm{~nm})$, since the human eye is much more sensitive to green than to red. One clearly sees in figure 5 that the fringes are shifted by one order of interference from the top to the bottom of the figure. The diffracted spot behind the cam thus carries orbital angular momentum with a $l=1$ topological charge. For a distance $d=3 \mathrm{~m}$, the topological charge is $l=2$, as expected. Besides, one can easily reverse the 2D asymmetrical object from a right to a left snail, just by turning it over. The interference pattern is reversed (see figure 5). The handedness of the diffracted laser beam is thus reversed to $l=-1$. Indeed one can simply generate a left-handed or a right-handed twisted beam with a plane wave and a 2D asymmetrical object just by turning it over. The diffracted twisted waves are thus chiral, with a size of the order of few micrometers. Recently, it has been suggested that such twisted waves could induce chirality $[29,30]$. As 
$2 \mathrm{D}$ objects and plane waves are not chiral whereas twisted beams are, the generation of chiral quantities (twisted beams) from non chiral quantities (diffraction of plane waves and asymmetrical 2D objects) may then shed some new light in the unsettling problem of the homochirality of life [31, 32].

We have thus been able to generate twisted beams from a linearly polarized plane wave diffracted by asymmetrical objects. We have checked numerically that the diffracted light from an inverted cam (the cam only is transparent), complementary to the cam we used, doesn't carry angular momentum in its shadow. There is thus no light carrying orbital angular momentum out of the cams shadow. We have also verified that the spin angular momentum has no influence since the polarization of the diffracted light is the same as the incident light. The angular momentum carried by the diffracted light from a cam is of orbital nature and lies in the shadow of the object, only. Then, due to the conservation of the angular momentum, the diffracting object must have acquired angular momentum. In principle, it should be possible to rotate asymmetrical $2 \mathrm{D}$ objects using plane waves.

To conclude, we have experimentally evidenced the generation of a twisted beam from the diffraction of a plane wave by a 2D simple asymmetric object. The topological charge corresponds to the symmetry of the diffracting object and is not conserved along propagation. One may now try to optimize the shape of the diffracting object depending on the applications. Such an experiment could be a way to generate twisted beams in a wide variety of lasers as in high power beams or pulsed lasers for example. Indeed, in high power instable cavity lasers, an intense Arago-Poisson spot arises in the shadow of the output mirror [33]. Using an asymmetrical output coupler would lead to a high power twisted beam behind the mirror. Besides, since the dark spot only slightly varies with the wavelength, one could create twisted beams on the whole spectrum of light with a single-easy-torealize diffracting object. Thus, pulsed lasers, or light electroluminescent diodes, 
or even white light twisted beams could be generated.

The authors thank L. Pruvost, D. Bloch, B. Viaris de Lesgno and C. Brousseau for valuable discussions, and J. R. Thébault, and A. Faisant for technical assistance.

* Corresponding author; olivier.emile@univ-rennes1.fr

[1] L. Allen, M.W. Beijersbergen, R.J.C. Spreeuw, and J.P. Woerdman, Phys. Rev. A 45, 8185 (1992).

[2] G. Molina-Terriza, J.P. Torres, and L. Torner, Nature Phys. 3, 305 (2007).

[3] M. Padgett and R. Bowman, Nat. Photonics 5, 343 (2011).

[4] F. Tamburini, B. Thidé, G. Molina-Terriza, and G. Anzolin, Nature Phys. 7, 195 (2011).

[5] B. Thidé, H. Then, J. Sjöholm, K. Palmer, J. Bergman, T.D. Carrozzi, Y.N. Istomin, N.H. Ibragimov, and R. Khanitova, Phys. Rev. Lett. 99, 087701 (2007).

[6] T. Kuga, Y. Torii, N. Shiokawa, T. Hirano, Y. Shimizu, and H. Sasada, Phys. Rev. Lett. 78, 4713 (1997).

[7] M.F. Andersen, M.E.J. Friese, N.R. Heckenberg, and H. Rubinsztein-Dunlop, Phys. Rev. Lett. 97, 170406 (2006).

[8] H. He, C. Ryu, P. Cladé, V. Natarajan, A. Vaziri, K. Helmerson, and W.D. Philipps, Phys. Rev. Lett. 75, 826 (1995).

[9] L. Paterson, M.P. MacDonald, J. Arlt, W. Sibbett, P.E. Bryant, and K. Dholakia, Science 292, 912 (2001).

[10] D.G. Grier, Nature 424, 810 (2003).

[11] K. Dholakia and T. Cizmar, Nat. Photonics 5, 335 (2011).

[12] J. Wang, J.Y. Yang, I.M. Fazal, N. Ahmed, Y. Yan, H. Huang, Y. Ren, Y. Yue, S. Dolinar, M. Tur, and A.E. Willner, Nat. Photonics 6, 488 (2012). 
[13] N. Bozinovic, Y. Yue, Y. Ren, M. Tur, P. Kristensen, H. Huang, A.E. Willner, and N. Ramachandran, Science 340, 1545 (2013).

[14] J. Leach, B. Jack, J. Romero, A.K. Jha, A.M. Yao, S. Franke-Arnold, D.G. Ireland, R.W. Boyd, S.M. Barnett, and M.J. Padgett, Science 329, 662 (2010).

[15] R. Fickler, R. Lapkiewicz, W.N. Plick, M. Krenn, C. Schaeff, S. Ramelow, and A. Zeilinger, Science 338, 640 (2012).

[16] C.E.M. Demore, Z. Yang, A. Volovick, S. Cochran, M.P. MacDonald, and G.C. Spalding, Phys. Rev. Lett. 108, 194301 (2012).

[17] J. Verbeeck, H. Tian, and P. Schattschneider, Nature 467, 301 (2010).

[18] M.W. Beijersbergen, L. Allen, H.E.L.O. van der Veen, and J.P. Woerdman, Opt. Comm. 96, 123 (1993).

[19] M.W. Beijersbergen, R.P.C. Coerwinkel, M. Kristensen, and J.P. Woerdman, Opt. Comm. 112, 321 (1994).

[20] N.R. Heckenberg, R. McDuff, C.P. Smith, and A.G. White, Opt. Lett. 17, 221 (1992).

[21] J. Liesener, M. Reicherter, T. Haist, and H.T. Tiziani, Opt. Comm. 185, 77 (2000).

[22] Z. Li, M. Zhang, G. Liang, X. Li, X. Chen, and C. Cheng, Opt. Express 21, 15755 (2013).

[23] O. Emile, A. Voisin, R. Niemiec, B. Viaris de Lesegno, L. Pruvost, G. Ropars, J. Emile, and C. Brousseau, EPL 101, 54005 (2013).

[24] J. Arlt, T. Hitomi, and K. Dholakia, Appl. Phys. B 71, 549 (2000).

[25] A. Jaouadi, N. Gaouloul, B. Viaris de Lesegno, M. Telmini, L. Pruvost, and E. Charron, Phys. Rev. A 82, 023613 (2010).

[26] D. Chauvat, O. Emile, M. Brunel, and A. LeFloch, Phys. Lett. A 285, 78 (2002).

[27] D.E. Dauger, Comput. Phys 10, 591 (1996).

[28] O. Emile, and J. Emile, http://arxiv.org/abs/1310.2444 (2013).

[29] Y. Tang, and A.E. Cohen, Science 332, 333 (2011). 
[30] C. Rosales-Guzman, K. Volke-Sepulveda, and J.P. Torres, Opt. Lett 37, 3486 (2012).

[31] S.F. Mason Nature 311, 19 (1984).

[32] J. Wang and B.L. Fering, Science 311, 1395 (2011).

[33] A.E. Siegman, Lasers University Science Books, Mill Valley (1990). 


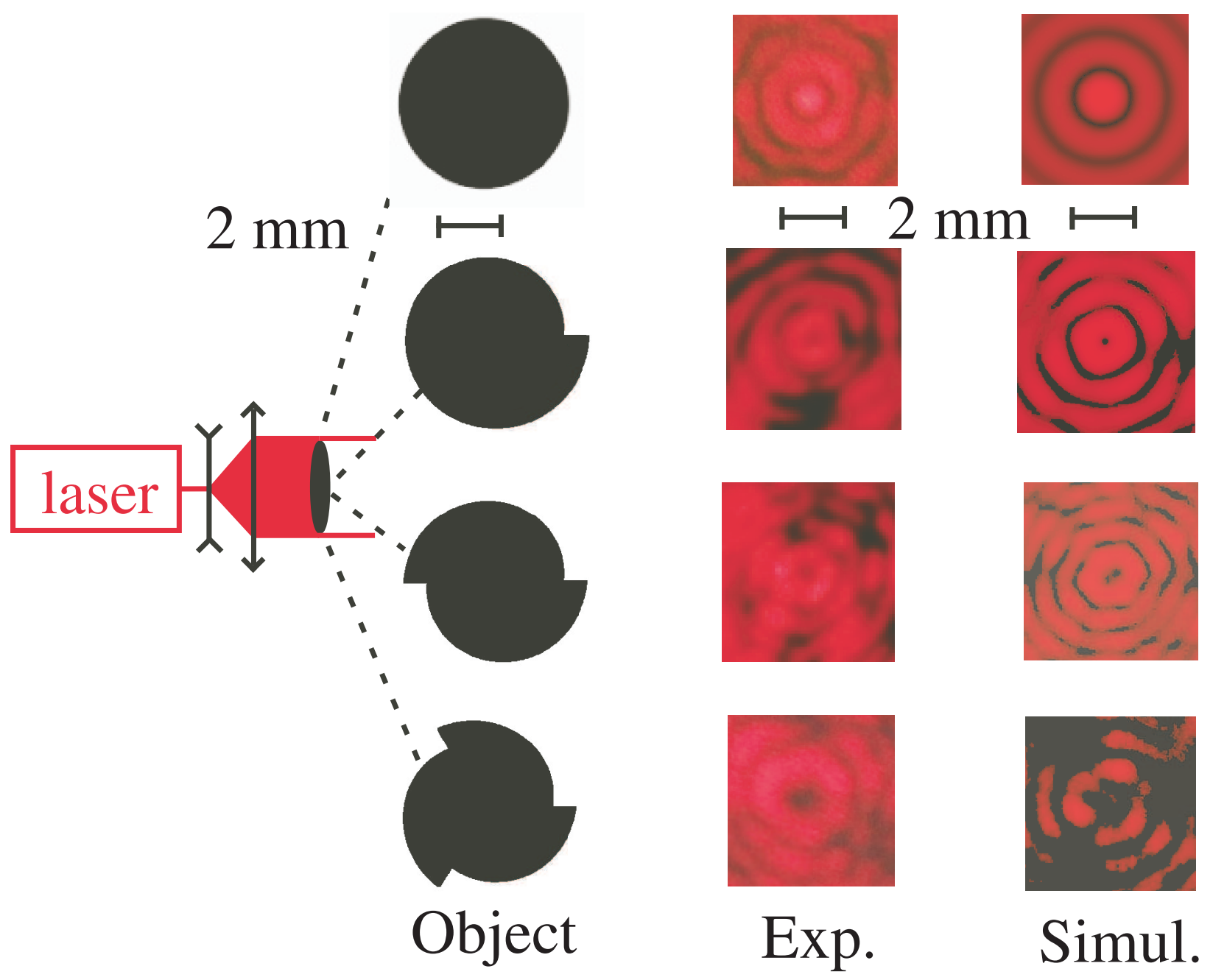

FIG. 1. Experimental and simulated diffracted beams. Diffracted pictures of a red laser beam diffracted by $2 \mathrm{D}$ objects recorded at a distance $d=6 \mathrm{~m}$ from the object. The red laser has been collimated using two lenses. In the case of a symmetrical object, one recognizes the well-known Arago-Poisson spot. In the case of an asymmetrical object, a strange hole appears in the diffracted bright spot in the shadow of the object. 

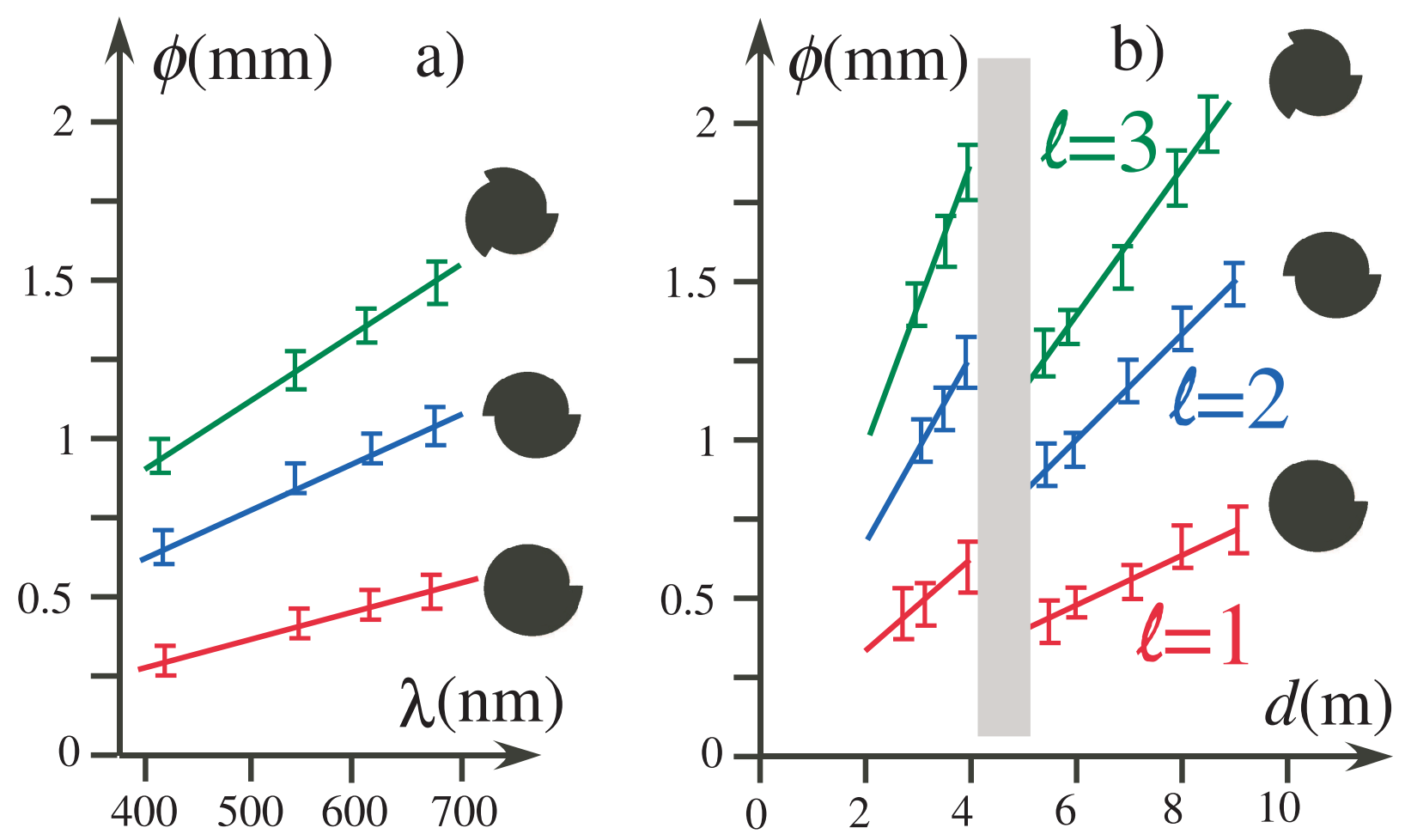

FIG. 2. Variation of the dark spot size. Variation of the diameter of the dark spot, a) versus the laser wavelength, for various asymmetrical $2 \mathrm{D}$ objects, and b) versus the distance between the diffracting object and the screen. The grey zone corresponds to distances where the dark spot is hardly noticeable. 

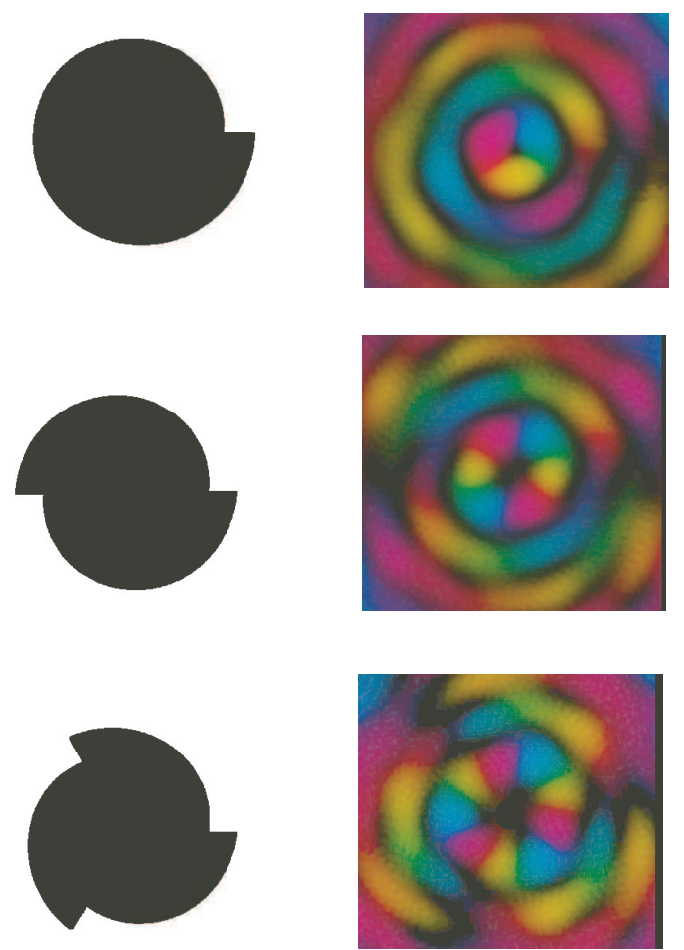

 \\ Object}
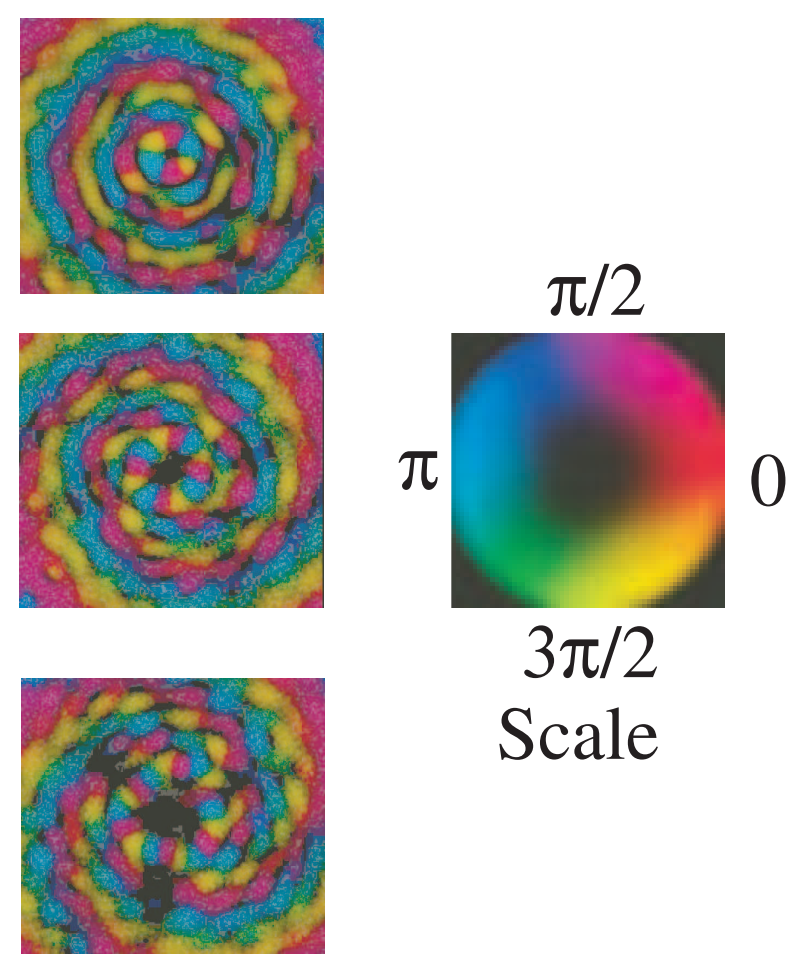

$3 \pi / 2$

Scale

FIG. 3. Simulated phase variation of the diffracted beam. Numerical simulations of the phase variation for three different asymmetrical diffracting $2 \mathrm{D}$ objects at two different distances $d$. The diffracted beam carries a topological charge with a value related to the symmetry of the diffracting object. 

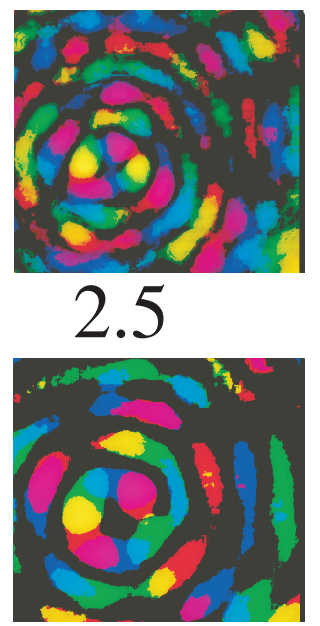

3.75

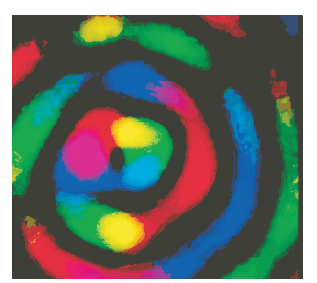

5.0

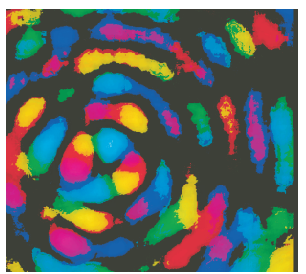

2.75
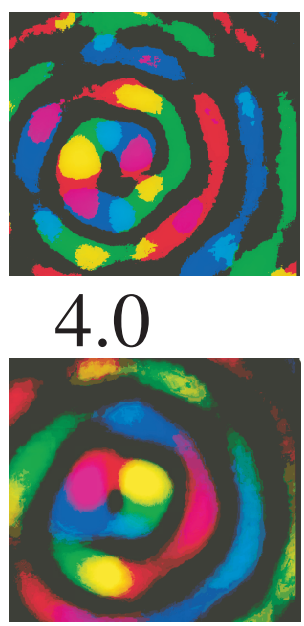

5.25

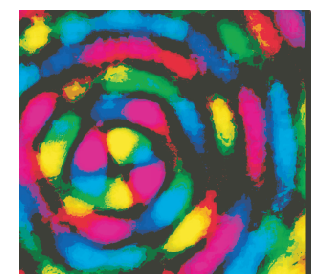

3.0
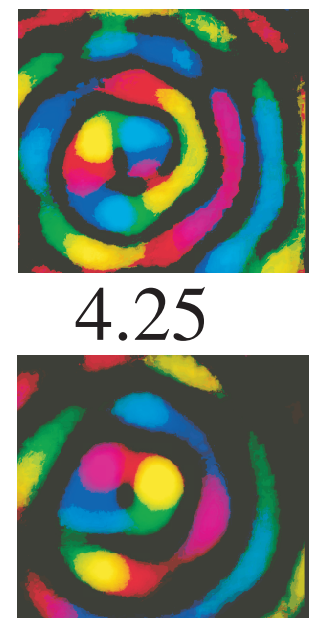

5.5

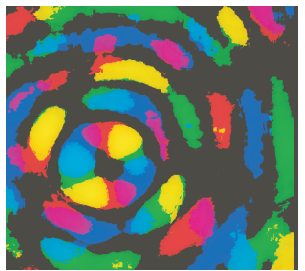

3.25

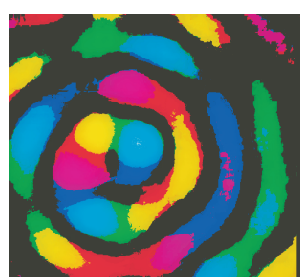

4.5

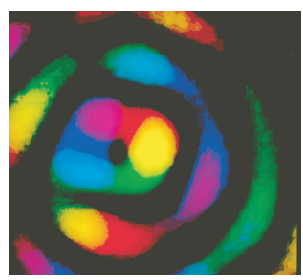

6.0

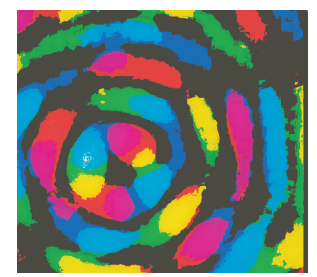

3.5



4.75

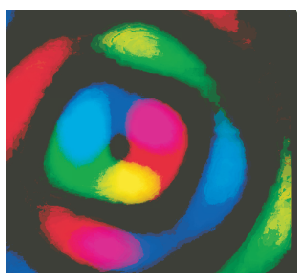

7.0

FIG. 4. Simulated phase variation of the diffracted beam for different distances $d$, from $d=2.5 \mathrm{~m}$ to $d=7 \mathrm{~m}$, in zone2, for a single snail shaped object. The topological charge varies from 2 for $d=3 \mathrm{~m}$ to 1 for $d=6 \mathrm{~m}$. The values below the pictures corresponds to the values of $d$ in meter. 

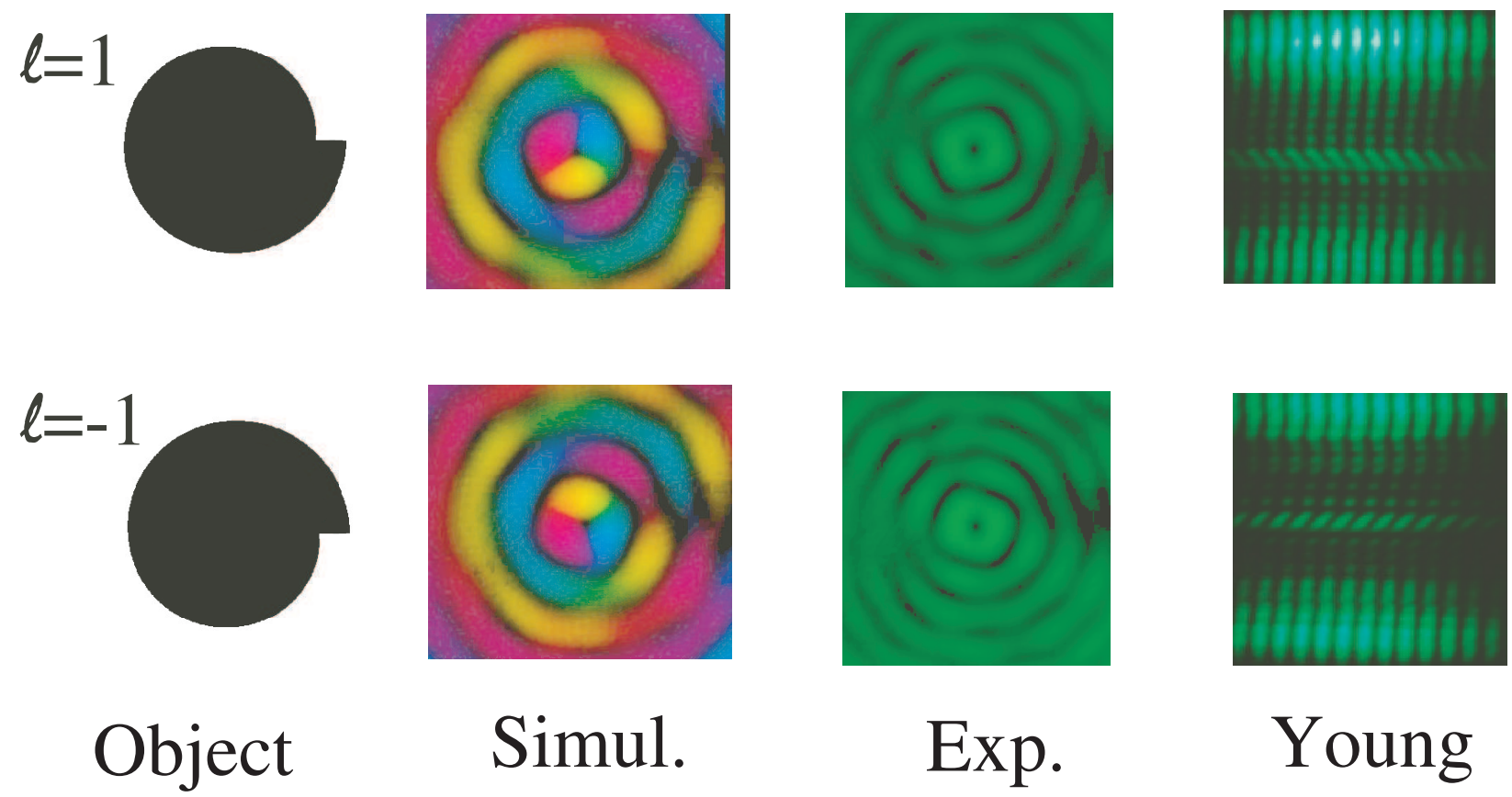

\section{Exp.}

\section{Young}

FIG. 5. Young's slit pattern of a diffracted green laser beam. Simulated phase variation of the diffracted beam by asymmetrical 2D objects, corresponding experimental beam intensity, and experimental diffraction pattern from Young's double slit set-up. Young's double slit is located $6 \mathrm{~m}$ after the diffracting cam. The slits are $50 \mu \mathrm{m}$ large and the distance between the slits is $200 \mu \mathrm{m}$. The interference patterns, observed at a distance of $4 \mathrm{~m}$ from the slits, correspond to 1 and -1 topological charges. 